\title{
La identidad en disputa. Conflictos alrededor de la construcción de la transexualidad
}

\author{
Gerard Coll-Planas \\ Universitat de Vic - Universitat Central de Catalunya. \\ Grup d'Estudis de Gènere: traducció, literatura, història i comunicació (GETLHIC) \\ gerard.coll@uvic.cat \\ Miquel Missé \\ Universitat Autònoma de Barcelona. Licenciado en Sociología
}

Recepción: 03-07-2013

Aceptación: 22-04-2014

\section{Resumen}

Como toda categoría identitaria, transexual tiene una definición normativa - en este caso, muy marcada por su origen médico-, hay unos agentes sociales que controlan su uso y existe un conflicto alrededor de los límites que abarca. En este artículo, analizamos cómo el conflicto por la delimitación de esta categoría se articula a nivel individual y colectivo. En base al análisis de entrevistas, grupos de discusión y mensajes en foros de Internet, se ha observado que hay un choque entre dos concepciones de la transexualidad. La primera defiende, desde postulados biologistas, que los criterios para entrar dentro de esta categoría son el cambio corporal y una identificación normativa con el género reivindicado. La segunda propone una interpretación más inclusiva y estratégica de la categoría. El choque entre estas dos concepciones permite entender el trasfondo de este debate aparentemente terminológico e invita a repensar la forma de entender la conexión entre el género y el cuerpo.

Palabras clave: género; transexual; transgénero; identidad de género.

\section{Abstract. Identity in dispute: Conflicts surrounding the construction of transsexuality}

'Transexual', like any other identity category, has a normative definition, which in this case is heavily influenced by its medical origin: there are social agents that control its use and there is conflict surrounding its boundaries. In this article, we analyze how the conflict over the definition of this category is managed through both individual and collective dimensions. Based on the analysis of interviews, focus groups, and Internet forum posts, it has been observed that there is a clash between two perceptions of transsexuality. The first one defends, from a biologist point of view, that the criteria to be included in this category are having undergone or wanting gender-related body modifications and following a normative reproduction of the claimed gender. The second one proposes a more open and strategic interpretation of the category. The clash between these two conceptions allows us to understand what is at stake in this apparently terminological debate and invites us to rethink the way we understand the connection between gender and body.

Keywords: gender; transsexual; transgender; gender identity. 


\author{
Sumario \\ 1. Introducción 4. Análisis \\ 2. Planteamientos teóricos 5. Conclusiones \\ 3. Trabajo de campo y método Referencias bibliográficas
}

\title{
1. Introducción
}

En el marco del postestructuralismo, se ha elaborado una crítica a la noción de la identidad como algo esencial y se ha puesto énfasis en el carácter construido de las categorías que utilizamos para designarnos (Foucault, 1976; Burr, 1996). Siguiendo esta línea de reflexión, se ha propuesto entender las categorías como constructos que son fruto de unas relaciones de poder determinadas. Estas reflexiones son especialmente pertinentes en relación con la categoría transexual, ya que tiene una definición normativa —en este caso, muy marcada por su origen médico-, hay unos agentes sociales que controlan su uso —-médicos, legisladores, las propias personas trans ${ }^{1}$, etc.- - y existe un conflicto alrededor de los límites que abarca (Weeks, 1985).

El objetivo de este artículo es analizar el conflicto alrededor del uso de la categoría transexual en contraposición a transgénero, tanto en el ámbito individual como en la acción colectiva. Este conflicto nos permite entender un debate, a menudo violento, dentro de la comunidad trans (Coll-Planas, 2010) y, al mismo tiempo, contribuye a reflexionar sobre la vinculación entre el género y la corporalidad.

A nivel español, las pocas aportaciones académicas en relación con el tema de la transexualidad han sido sobre todo en el ámbito del derecho y de las ciencias de la salud. Desde las ciencias sociales, se ha analizado la situación de las personas trans en ámbitos específicos como la educación (Casanova, 2011; Fontana y Ferriols, 2011; Hinojosa, 2011; Lozano, 2011; Platero, 2007), los medios de comunicación (Carratalá, 2011; Marini et al., 2010), en relación con los efectos sociales de la legislación (Belsué, 2012; Rubio, 2008; Soley-Beltran, 2007) y en las políticas públicas (Platero, 2009). Sin embargo, el ámbito que ha despertado más interés es el médico (Coll-Planas y Missé, 2012; Martínez e Î́niguez, 2010; Martínez y Montenegro, 2010; Nieto, 2006; Tena, 2013). En este campo, se ha estudiado el discurso médico alrededor de la transexualidad, el modo como se concreta el acompañamiento médico a las personas trans en un marco patologizador y los discursos críticos del activismo. Otro aspecto que se ha abordado ampliamente es el estudio de la transexualidad desde una vertiente más teórica, para explicar qué función tiene

1. En este artículo, se utiliza trans como término que engloba a todas aquellas personas que no se sienten identificadas con el género atribuido al nacer en función de su sexo. Así pues, no distingue si la persona se ha operado o el sentido político que atribuye a su identidad de género. 
en relación con el género normativo y cómo las vivencias de las personas trans contribuyen a reproducir y a desplazar las normas del género (Balza, 2009; Belsué, 2011; Juliano, 2004, 2010; Martínez y Montenegro, 2011; Rodríguez, 2001; Soley-Beltran, 2009; Vartabedian, 2007). En relación con el tema del presente artículo, la construcción de la identidad transexual en diálogo con la categoría transgénero, los trabajos que más se acercan son los que han analizado el impacto de la migración en mujeres trans provenientes de Latinoamérica, pues muestran el choque entre la identidad transexual y la travesti (García y Oñate, 2010; Vartabedian, 2008, 2012).

Más allá de nuestro contexto, el tema de nuestro estudio ha sido abordado por diversos autores y autoras en el ámbito norteamericano, en el marco de los denominados transgender studies. Las principales aportaciones han planteado cómo la categoría transexual ha sido construida por los discursos médicos y cómo las propias personas que cambian de sexo y/o de género han ido cuestionando y modificando su significado (Billings y Urban, 1983; Stone, 1991; Feinberg, 1992; Hausman, 1998; Stryker, 2008; Valentine, 2007). En este marco, se han desarrollado reflexiones críticas en relación con la categoría transexual y se han propuesto terminologías y discursos que abren la forma de entender la cuestión del cambio de sexo y/o de género, como son las categorías queer y transgénero. Nuestra aportación consiste en ir un paso más allá, es decir, no solo analizar los efectos de la categoría transexual, sino también estudiar estos nuevos paradigmas para mostrar cómo estas categorías, aparentemente más inclusivas, también pueden configurar nuevas exclusiones.

A continuación, abordamos los aspectos teóricos que nos permitirán analizar el conflicto alrededor de la definición y los límites de la categoría transexual. En el apartado 3, presentamos el diseño de la investigación y el trabajo de campo. En el siguiente apartado, analizamos los usos y los conflictos de esta categoría en el plano individual y en el activismo. Acabamos el artículo con unas conclusiones en las que nos proponemos dar cuenta de las diferentes posiciones en relación con el debate y de las consecuencias teórico-políticas que se derivan de ellas.

\section{Planteamientos teóricos}

Este apartado está dividido en tres secciones. En primer lugar, explicitamos nuestra forma de entender las categorías identitarias. En segundo lugar, desde esta perspectiva, damos cuenta de la aparición de la categoría transexual en el marco del ámbito médico. En tercer lugar, contextualizamos la recepción y el uso de la categoría transexual en el caso español.

\subsection{Concepción de las categorias identitarias}

En el marco del debate teórico de la sociología del género, se produce una confrontación entre entender la identidad como algo esencial o como algo construido socialmente. El esencialismo supone que existe una esencia real, 
auténtica, de aquello que es inmutable y constitutivo de una persona o cosa, a menudo apelando a argumentos biologistas (Goldberg, 1973; Fisher, 2000). Dicha esencia se considera presocial: la organización social la puede fomentar o reprimir, pero no modificar (Fuss, 1999).

En contraposición, la perspectiva que adoptamos en este artículo entiende que las categorías son construcciones históricas constituidas en el marco de relaciones de poder (Foucault, 1976; Weeks, 1985). Las categorías identitarias, además, son performativas: no describen la realidad de los sujetos que designan, sino que producen su subjetividad (Butler, 1990, 1997). Las categorías, en este sentido, tienen un doble efecto de restricción y de producción de posibilidades.

El establecimiento de categorías identitarias resulta ambivalente, pues estas oprimen al sujeto y, al mismo tiempo, le dan vida. En esta línea, siguiendo a Hegel, Butler (1997: 14) afirma que «cualquiera de nosotros se constituye como ser social viable únicamente a través de la experiencia del reconocimiento». Sigue, además, una concepción foucaultiana del poder, no como una entidad que presiona al sujeto desde fuera y de una forma restrictiva, sino como un elemento que funda al sujeto, le proporciona existencia y le confiere identidad. En este sentido, las normas sociales dan vida a los sujetos y, al mismo tiempo, los oprimen, como recoge el concepto de sujeción, proceso por el cual nos convertimos en sujetos al someternos al poder (Butler, 1997).

Así, la necesidad de pertenecer a la sociedad implica tratar de reconocerse y de ser reconocido en sus categorías. La misma noción de humano está presa por categorías como el género, y es impensable o monstruoso alguien sin un género identificable, como muestran los obstáculos con que se encuentran las personas intersexuales (Cabral, 2009) o las personas trans que no reproducen los roles masculino y femenino (Coll-Planas, 2010).

\subsection{La construcción de la categoría transexual}

Para abordar la génesis de la categoría transexual, es preciso retrotraernos al siglo XVIII, cuando se establece la categoría homosexual (Foucault, 1976; Weeks, 1981). Un eje central en la definición de homosexual fue su asociación con la inversión de género: se consideraba que su «anomalía» no era su objeto de deseo (una persona del mismo sexo), sino el hecho de desear desde una posición «invertida» (una mujer que desea como un hombre y viceversa en el caso del hombre homosexual). Así, la homosexualidad se entendía como una especie de «androginia interior, de hermafroditismo del alma» (Foucault, 1976: 57-8).

En los círculos médicos, la distinción entre atracción por parte de personas del mismo sexo e inversión de género se empieza a producir a finales del siglo XIX, cuando, desde la sexología, se acuña la categoría clínica travestismo. Havelock Ellis y Magnus Hirschfeld encabezan la campaña para establecer travestismo y homosexualidad como categorías separadas (Llamas, 1998). Defienden que sentirse atraído por personas del mismo sexo no está necesariamente asociado a travestirse, al amaneramiento y a sentirse del sexo contrario y, al 
mismo tiempo, que alguien se puede travestir manteniendo una tendencia heterosexual (King, 1998).

La distinción entre las personas con comportamientos puntuales con fines sexuales y las que se identifican permanentemente con el sexo contrario y quieren cambiar sus características sexuales se produce en la década de 1950, cuando aparece el concepto de transexual, acuñado por David Cauldwell y popularizado por Harry Benjamin (Llamas, 1998). La palabra transexual hace referencia a aquella persona que se siente del sexo contrario y desea modificar su cuerpo quirúrgicamente para parecerse a las personas del sexo opuesto (King, 1998). La aparición de esta categoría está determinada por las posibilidades técnicas de llevar a cabo este tipo de operaciones. Resulta clave, en este sentido, el desarrollo de la endocrinología y la cirugía plástica, que no solo ofrecen las técnicas para hacer posible la operación de reasignación sexual, sino que también establecen un campo simbólico que hace concebible la noción de transexualidad (Hausman, 1998). A partir de la década de 1970, se produce una creciente aceptación de la cirugía de reasignación sexual dentro de los círculos médicos y empieza a institucionalizarse. En este período, se consolida el término transexual, mientras que el de travestido prácticamente desaparece del ámbito médico (King, 1998).

\subsection{La adopción de la categoría transexual en el contexto español}

A continuación, analizamos el impacto que ha tenido la categoría transexual en el contexto español. Para hacerlo, nos basamos en un artículo previo (SoleyBeltran y Coll-Planas, 2011) que compara la situación de la transexualidad a partir de dos estudios: el primero, llevado a cabo entre 1998 y 2000 (Soley-Beltran, 2005, 2007) y el segundo, en 2008 (Coll-Planas et al., 2009; Coll-Planas, 2010). En base a esta comparación, observamos que, a lo largo de la última década, se ha producido una creciente medicalización de la transexualidad, lo que ha revertido en un cambio en el uso de las categorías identitarias.

En el primer estudio, las personas entrevistadas muestran una visión de la medicalización como un avance frente al imaginario anterior respecto a la transexualidad, conservador y moralizante (Pérez, 2010). En el estudio llevado a cabo diez años más tarde, en cambio, se observa una relación mucho más ambivalente respecto al estamento médico: por un lado, aparece como un aliado que posibilita los tratamientos que demanda parte de la comunidad trans; por otro, se observa una creciente crítica a la patologización de la transexualdad (etiquetada como trastorno por la Organización Mundial de la Salud y la Asociación de Psiquiatría Americana), así como a los procesos y a los criterios médicos que rigen la obtención del certificado de trastorno mental (Missé y Coll-Planas, 2010).

Esta creciente medicalización está respaldada por la legislación que regula el cambio de mención de sexo de los documentos oficiales (requisito necesario para poderse cambiar el nombre). La Ley 3/2007, de 15 de marzo, establece que, para poder efectuar este cambio, es preciso presentar un certificado conforme la persona presenta el trastorno de «disforia de género» y otro acreditan- 
do que se ha sometido a un tratamiento médico, de como mínimo dos años, para modificar sus características sexuales (Belsué, 2012; Coll-Planas y Missé, 2012; Missé, 2013).

La fuerza de la medicalización y la nueva legislación son los elementos clave que explican el cambio que se ha producido en relación con el uso de las categorías identitarias dentro de la comunidad trans española. En 1998, el término transexual era usado solamente por las personas trans activistas y que estaban más informadas, mientras que el término socialmente más utilizado era el de travesti. En la década de 1990, la distinción entre transexuales preoperados y postoperados, muy usada en los países anglosajones, era mucho menos relevante en España: muchas transexuales femeninas, por ejemplo, feminizaban su apariencia mediante tratamiento hormonal, cirugía o a través del vestido, pero mantenían sus genitales masculinos (Soley-Beltran, 2005).

En la actualidad, por un lado, la categoría transexual se ha institucionalizado, en gran parte, debido a la creciente influencia médica, sancionada por la legislación. Por otro lado, han proliferado otras categorías identitarias, como trans, queer, genderqueer, transfeminista o transgénero. Esta última ha vivido una evolución diferente a la experimentada en el contexto anglosajón (Valentine, 2007), mientras que el término transgender ha perdido su connotación política en el contexto anglosajón, para convertirse en una designación paraguas de las personas que no se sienten del género asignado en función de su sexo. En España, funciona como un término que denota una voluntad de alejarse del paradigma dominante de entender el género, considerado patologizador, binarista y reificador de las categorías masculino y femenino.

\section{Trabajo de campo y método}

Para analizar la dimensión individual de la problemática, nos hemos basado en tres técnicas: entrevistas, grupos de discusión y recopilación de mensajes de Internet. El trabajo de campo tuvo lugar en Cataluña, entre septiembre de 2007 y enero de 2008.

En primer lugar, se realizaron ocho entrevistas semidirigidas: cuatro a trans femeninas y cuatro a trans masculinos. Las entrevistas duraban, de promedio, una hora y media. Los nombres de las personas entrevistadas han sido cambiados por pseudónimos. En segundo lugar, se llevaron a cabo dos grupos de discusión, que duraron cuatro horas: una hora y media de debate en dos subgrupos (trans femeninas y trans masculinos) y, después de una pausa, una hora y media de debate conjunto. En el primer grupo, participaron seis personas y en el segundo, cinco. En tercer lugar, se compilaron 600 mensajes de dos foros de Internet: uno de trans masculinos y otro de trans femeninas. Se escogieron estos foros de debate por ser los más activos de los destinados a dichos colectivos en el contexto español. Se seleccionaron todos los mensajes relacionados con la transfobia y con la vivencia del género sentido.

En relación con el trabajo de campo sobre el activismo, se ha seguido la lógica del estudio de caso paradigmático (Flyvbjerg, 2004), que parte de 
la selección de casos que ponen de relieve las características generales de la problemática estudiada. Siguiendo esta lógica, se han seleccionado dos artículos publicados en el portal Dosmanzanas, que, con una media aproximada de 3.200 visitas al día ${ }^{2}$, es el espacio virtual de información y opinión de referencia del colectivo LGTB a nivel español. Después de realizar una exploración del conjunto de entradas en relación con el tema de la transexualidad, se escogieron las dos que han recibido más comentarios (177 en total), lo que muestra su impacto y relevancia. Ambos artículos y los comentarios recibidos, que también son objeto de análisis, permiten estudiar cómo el debate alrededor de la identidad trans se traslada al ámbito de la acción colectiva, pues las autoras de ambos artículos son miembros destacados de asociaciones del colectivo transexual.

El primer artículo es «La transexualidad no es queer» (en adelante, «Art1»), de Mar Cambrollé3, que fue publicado el 18 de octubre de 2008 y recibió 102 comentarios. El segundo es «Octubre Trans? Red por la despatologización? Neotransfobia? No en mi nombre» (Art2), de Ainara Ruiz ${ }^{4}$, que fue publicado el 26 de octubre de 2010 y recibió 75 comentarios.

En las referencias a las citas del trabajo de campo, especificamos primero la fuente: Ent (entrevista), Foro, GD (grupo de discusión), Art (artículos analizados de la vertiente de la acción colectiva) y Com (comentario en respuesta a los artículos). A continuación, explicitamos si la autoría corresponde a una trans femenina (TF) o a un trans masculino (TM). Además, hay que señalar que los mensajes de los foros, los artículos y los comentarios de los mismos se presentan en su forma original, únicamente se han corregido los aspectos ortográficos.

\section{Análisis}

\subsection{Nivel individual}

De las diferentes posiciones que adoptan las personas trans, empezaremos examinando las que, con el biologismo de fondo, defienden la necesidad de realizar un cambio corporal para considerarse plenamente transexuales: «Si el cerebro en el feto se forma como mujer, tiene una necesidad biológica, de nacimiento, de verse como tal» (GD2, TF). Este tipo de afirmaciones refuerza la normatividad de la correspondencia entre sexo y género, al considerar que una persona que «verdaderamente» se siente mujer, «lógicamente» quiere tener unos genitales "femeninos» (y viceversa en el caso de las personas que se sienten hombres): «Si realmente eres transexual, lo más probable es que quieras hacer tu vida como una persona del sexo opuesto [...] y estás dispuesto a hacer la transición sin ninguna excusa» (Foro, TF).

2. http://www.dosmanzanas.com/informacion (consultada el 15 de julio de 2013).

3. http://archivo.dosmanzanas.com/index.php/archives/5697 (consultada el 15 de julio de 2013).

4. http://www.dosmanzanas.com/2010/10/octubre-trans-red-de-despatologizacion-neotransfobia-no-en-mi-nombre.html (consultada el 15 de juliol de 2013). 
El manual de clasificación de enfermedades mentales de la Asociación de Psiquiatría Americana, el DSM-V, de hecho, consagra la aversión a los genitales como uno de los criterios para establecer que una persona experimenta disforia de género. Esto alimenta la sensación de tener que rechazar sus genitales originales y provoca que las personas trans que disfrutan de ellos tiendan a esconder esta información a sus médicos (Coll-Planas, 2010; Soley-Beltran, 2005).

La discusión sobre la necesidad de realizar la reasignación sexual se produce más intensamente entre trans femeninas, lo que se puede deber a dos motivos:

- En primer lugar, la operación para los trans masculinos no está bien resuelta estéticamente, no se financia públicamente y produce más problemas de salud, por lo que muchos no se someten a ella.

- En segundo lugar, socialmente la presencia de pene cuestiona fuertemente la feminidad de las mujeres trans, mientras que la ausencia del mismo no pone tan en entredicho la masculinidad de un hombre trans:

[En] gran hermano / hace unos años / entró un transexual masculino / y nadie le preguntó qué genitales tenía o dejaba de tener, se le trató con respeto absoluto, y nadie dijo que no fuera un hombre / este año entró una transexual femenina / y ha pasado lo contrario [...] se cuestiona continuamente la feminidad de la concursante [...] Mercedes Milá / ha dicho en directo que amor «tenía pene» y aun era un hombre. (Ent. Andrea, TF)

Esto se puede deber al hecho de que, en nuestra cultura, la mujer se entiende más cercana a la naturaleza y a lo corporal (Weeks, 1985; Juliano, 2004), mientras que el hombre se concibe más cercano a la cultura, más ajeno a la naturaleza, por lo que las características de su cuerpo no son tan importantes como lo que hace con él, su agencia.

Las personas trans que sostienen la necesidad de mantener la correspondencia entre sexo y género efectúan una exclusión de quien se define como transexual sin haberse operado: «Te reivindicarás mujer con pene, pero eso no existe ni en el circo» (Foro, TF).

Si profundizamos en este aspecto del discurso transexual, observamos que la inclusión o la exclusión en la categoría no tiene que ver, efectivamente, con el hecho de si la persona se ha operado o no los genitales, sino con si siente rechazo hacia ellos y querría operárselos. La transexualidad, entonces, no tiene que ver con algo observable (tener los genitales de origen o haberse operado), sino con una forma de entender su identidad de género:

La realidad es que te puedes encontrar personas con pechos y con pene (a priori, transexuales femeninas sin reasignar), pero que, en realidad, son de dos tipos diferentes [...]: tipo A: mujeres con polla; tipo B: hombres con tetas.

5. Esta entrevista se realizó en línea a través de un programa de mensajería instantánea. Utilizamos la barra inclinada para marcar el momento en que la entrevistada saltaba de línea en el chat. 
Aunque desnudas parezcan lo mismo, lo cierto es que no tienen nada que ver. (Foro, TF)

En este sentido, encontramos personas trans que, a pesar de defender la necesidad de mantener la correspondencia entre sexo y género, contemplan algunos motivos para, considerándose transexuales, no llevar a cabo la reasignación sexual: que el resultado de la operación no es el esperado («sabe que lo que le harían siempre iba a ser solo una chapuza»; foro TM), por motivos de salud («si alguien no puede operarse porque está enfermo de algo y si se opera se puede morir, pues es aceptable»; foro TM) o por motivos económicos. Nótese que estas aportaciones adoptan un tono normativo (sobre las objeciones que son «aceptables» para no operarse, sobre quién «debería» considerarse transexual, etc.), lo que constituye un ejemplo del proceso de definición normativa de las categorías identitarias.

Las personas que se definen como transexuales sin querer una reasignación sexual denuncian el «racismo transexual» que pretende dividir el colectivo entre «auténticas» y «falsas» transexuales (Foro, TF). Defienden, además, la compatibilidad entre ser transexual y no sentir aversión hacia (o incluso disfrutar con) los genitales originales, como afirma este trans masculino: «Si a mí me viene una persona a contarme que se siente hombre pero que está muy a gusto con sus genitales femeninos y no los cambiaría por nada del mundo, yo no me siento quién para "autorizar" a esa persona a que "sea hombre" o lo deje de ser» (Foro, TM). En la misma línea, una chica trans cuestiona la necesidad de rechazar los genitales: «Si una tiene un medio a su alcance para proporcionar y proporcionarse placer, es lógico que lo utilice» (Foro, TF). Desde esta posición, se critica el requisito de rechazo genital:

Hay por ahí «médicos» sentenciando que no operarían una reasignación de sexo a alguien que se haya masturbado alguna vez [...] hay personas que logran salir del bloqueo mental que les producen sus genitales y utilizarlos completamente, creo que eso es sano para ell@s. Tienen un cuerpo que pueden utilizar. (Foro, TM)

En esta línea, desde el discurso transgénero, se cuestiona la necesidad de realizar cambios corporales y de reproducir un rol de género, lo que a menudo provoca «rechazo" por parte de los «trans normativos» (GD1, TM). En palabras de un chico transgénero: «Podemos tener puntos de desacuerdo muy fuertes, como si puedo o no puedo quedarme embarazado, si debo o no debo hacerlo, si es ético o inmoral» (GD1, TM).

Este tipo de planteamientos cuestiona lo que define a un hombre y a una mujer, desvinculando el cuerpo (sexuado como macho o hembra) y la identidad de género (hombre o mujer): «He visto chicos súper masculinos, con su pecho, y con la camisa desabrochada y totalmente tranquilos, cada uno es lo que es por lo que quiera ser» (Ent. Álex, TM). Esto supone reconceptualizar la vinculación entre cuerpo y género hasta el punto de cuestionar que los pechos, la vagina, la menstruación o el embarazo sean elementos inherentemente femeninos, o que tener pene sea algo necesariamente masculino. 
El discurso transgénero, como volveremos a ver en el próximo apartado, crea una fuerte discrepancia por parte de las personas que reproducen el discurso transexual, y es que el primero cuestiona las bases en que se funda el último: se distancia del biologismo determinista afirmando que hay margen para la agencia, cuestiona la idea de que el proceso transexualizador sea irreversible y, finalmente, quiebra la visión normativa del género, al afirmar que se puede vivir al margen de la masculinidad y la feminidad.

Así pues, ambos discursos chocan, porque tienen estrategias y fines políticos contrapuestos. Desde el discurso transgénero, la clave es desvincular el género y la corporalidad y no reproducir la masculinidad ni la feminidad de forma normativa. En contraposición, según las personas que defienden la necesidad de realizar la modificación corporal, la integración social se conseguiría «gracias a personas que demuestran que un cambio completo y real es posible, sin medias tintas y sin pasar por monstruitos raros» (Foro, TF).

\subsection{Nivel activista}

En este apartado, analizamos cómo se traslada el choque entre los discursos transexual y transgénero en el activismo. Las autoras de los dos artículos que son objeto de estudio y que dieron lugar a prolíficos debates se pueden enmarcar en el discurso transexual. En ambos casos, la modificación corporal se considera central en la definición de la transexualidad:

Tod@s hemos realizado todo lo que hemos podido por adecuar nuestro físico al sexo sentido: tratamientos hormonales (sin control y automedicándonos, con el riesgo que ello implica para la salud), cirugías de feminización del rostro, mamoplastias, etc. Todo ello [...] con el mayor y más profundo deseo de percibirse y de que te perciban como mujer u hombre. (Art1)

Esta defensa del cambio corporal para poder denominarse transexual tiene sus excepciones. La autora del artículo 1, por ejemplo, está de acuerdo en que la legislación vigente contemple que puedan acceder al cambio registral sin necesidad de realizar ningún tratamiento personas que no puedan operarse "por motivos de salud o riesgo para sus vidas» (Art1). Sin embargo, se muestra contraria a que se suprima la necesidad de seguir un tratamiento en los demás casos. Estas excepciones, como hemos visto en el ámbito individual, indican que el cambio corporal en sí mismo no es condición única (ni tan solo necesaria) para considerarse transexual, pero sí el rechazo y la voluntad de modificar el cuerpo.

En los artículos y en parte de los comentarios que los respaldan, se reproduce el discurso transexual que sostiene una concepción esencialista y una reivindicación de las identidades femenina y masculina. Esta reivindicación, sin embargo, no implica una negación de la realidad de las personas que no se sienten ni hombres ni mujeres: «Respeto totalmente su identidad, pero son realidades diferentes» (Com70, Art1). En este sentido, se establece una clara distinción entre transexual y transgénero como dos categorías identitarias diferenciadas. Esta voluntad de diferenciación deriva en un rechazo del tér- 
mino paraguas trans, que se ve como una negación de sus realidades y de sus necesidades específicas, considerando que lleva a «invisibilizar y distorsionar la realidad de las personas transexuales a través de una etiqueta trans, en la que se mete cualquier cosa muy diferente de las personas transexuales, con necesidades y reivindicaciones totalmente diferentes» (Art1). Esta crítica al concepto trans por pretender amalgamar elementos muy distintos entre sí se ha llegado a formular, de forma sarcástica, con la expresión: «Las personas trans, transexuales, transgénero, trans...portistas de cabras y demás» (Com31, Art1).

La incomodidad, sin embargo, no se debe solamente al hecho de pretender unir realidades diferentes, sino a que parte del colectivo transexual siente como un ataque los discursos de las personas que se autodenominan transgénero o queer: "Todo eso solo tiene un nombre: neotransfobia (un ataque a nuestra identidad y derechos tan brutal como la transfobia "clásica», pero de forma más sibilina y sistemática, camuflada de una supuesta lucha personas trans [sic]» (Art1). Veamos, a continuación, como se argumenta que las personas transgénero atacan a los derechos de las transexuales.

En primer lugar, porque sienten que el cuestionamiento del binarismo de género les está negando su identidad como mujeres y como hombres transexuales. Dos elementos clave para entender este conflicto son la demanda de la red STP 2012, que lidera la campaña por la despatologización de la transexualidad (ver Missé y Coll-Planas, 2010), de eliminar la mención de sexo del DNI y el lema "Ni hombres ni mujeres, el binarismo nos enferma», que se utilizó en 2008 en manifestaciones por la despatologización en distintas ciudades españolas. La autora del artículo 1 rechaza este lema, argumentando que «si algo tenemos claro las personas transexuales es que deseamos ser mujeres y hombres». Esta crítica se elabora, pues, desde la posición de gente que ha luchado y está luchando para ser reconocida en función de su identidad de género.

En la siguiente cita, además, se apunta que la posición no binaria perjudica la aceptación social:

Me parece muy respetable que ell@s quieran ser libres en su plenitud, que sean hombres y mujeres hoy sí, mañana no, pasado quién sabe cómo se identificarán, pero no dejaré que me tachen de "desviado» o «loco» por el hecho de que no sepan como identificarse claramente. (Com39, Art1)

En segundo lugar, se critica la noción de la identidad como una construcción social, la idea de que "nuestra identidad es un invento occidental, de los médicos estadounidenses de los años cincuenta» (Art1). De hecho, esta es una interpretación sesgada de un documento de la red española STP2012 , que decía:

La transexualidad es un término producido por la medicina norteamericana en la década de 1950, es una palabra pensada para categorizar y etiquetar las

6. Guia de buenas prácticas para la atención sanitaria a personas trans en el marco del sistema nacional de salud. Fuente: http://stp2012.files.wordpress.com/2010/10/stp-propuestasanidad.pdf (consultado en julio de 2013). 
trayectorias vitales de aquellas personas que han nacido con un cuerpo de hombre pero viven en femenino y las personas que han nacido con un cuerpo de mujer pero viven en masculino.

Consideramos que esta lectura de la argumentación construccionista es clave para entender cómo, desde el discurso transexual, se recibe con dolor e incomprensión la defensa de que la categoría que sienten que les define no es algo esencial, sino construido socialmente. Esta consideración se entiende como una falta de respeto hacia las personas trans que sienten su identidad como una esencia y que han tratado de cambiarla antes de aceptarse:

Una cosa es que los roles de género sean culturales y otra cosa es eso de que la «identidad sexual sea una imposición cultural». La realidad es más bien al contrario, con toda la presión cultural y social que sufrimos desde pequeñitas y pequeñitos que nos dice que somos del sexo al que corresponden nuestros genitales (y nuestros cromosomas y nuestros cuerpos en general), desde la familia, amistades, educación, medios de comunicación, etc. que, claro, las personas transexuales a pesar de todo ello, tenemos clara nuestra identidad sexual y sabemos que, al contrario de todo lo que nos dicen (e imponen), no somos del sexo de nuestros genitales, sino del otro. (Com36, Art1)

En la argumentación de este punto, se mezcla la idea de la identidad como construcción social con el voluntarismo, posiciones que no tienen por qué ir de la mano: como hemos planteado en el apartado teórico en base a Butler (1990; 1997), entender que las categorías identitarias son construidas, no implica considerar que sean modificables a voluntad del individuo, sino que se enraízan profundamente en nuestra subjetividad, con lo cual se reduce nuestro margen de agencia (Coll-Planas, 2010, 2012).

En tercer lugar, se critica que las personas transgénero y queer ataquen su demanda de atención sanitaria por «el continuo desprestigio de las soluciones y los tratamientos que necesitamos las personas transexuales (como son la hormonación y las cirugías), presentándolas como una "imposición de la sociedad y la clase médica" [sic] [...] y como una "agresión a nuestro cuerpo" [sic]" (Art1). Otro ejemplo que cita la misma autora en un comentario posterior es el hecho de tildar de "macabras operaciones» las cirugías de reasignación de sexo (Com70, Art1).

Para las personas que se expresan en el debate virtual desde una posición transexual, el problema no es solo que el discurso transgénero contradiga los fundamentos en que se basa la categoría transexual, sino que, mediante la utilización de la categoría trans, se trate de usurpar la voz del conjunto del colectivo. Esto, además, se agrava porque se considera que, en las filas transgénero, se unen personas no trans («se mezclan feministas radicales, movimientos universitarios, etc.»; Com2, Art2) que acaban apoderándose de la voz de las «verdaderas» personas transexuales. En varios momentos (por ejemplo, en Art1), aparece la sensación de que activistas no trans manipulan a las personas transexuales con sus propios fines, hablan en su nombre y los utilizan para 
promover demandas queer que van en contra de los intereses de las personas transexuales.

En este sentido, desde el discurso transexual, las personas transgénero son construidas como personas privilegiadas y alejadas de la realidad: «No podemos permitir que una pandilla de fiesteros universitarios con resaca representen a un colectivo tan castigado por la sociedad como es el nuestro" (Com25, Art1). En la misma línea, encontramos también las opiniones manifestadas en: Com95, Art1; Com39, Art1, y Com45, Art1. Un chico gay que interviene en el debate acusa a las personas transgénero de actuar con autoritarismo:

Tratáis a las personas transexuales como si fuesen "víctimas de la sociedad», engañadas y manipuladas por la presión social para ser algo que no tendrían por qué ser. Vosotros y vosotras, las personas transgéneros, sois como más listas: os habéis liberado de los yugos, habéis visto la luz. Las personas transexuales son como las primas tontas de pueblo, que os acompañan, que incluso habláis por ellas, porque no se enteran y sabéis mejor que ellas lo que les conviene. Defendéis la despatologización pero las tratáis como si fueran enfermas mentales. (Com44, Art1)

Una vez tratados los argumentos de las personas que sostienen un discurso transexual, analicemos las posiciones de las que participaron en el debate criticando este tipo de argumentos desde un discurso transgénero o queer.

Por lo que al cambio corporal se refiere, la mayoría de los comentarios defienden que las personas que quieran modificar su cuerpo tengan derecho a hacerlo, pero reconociendo también como válidas las trayectorias de las personas que no optan por este tipo de cambios:

Muchas personas transexuales se hormonan y, sin embargo, no consideran ni de lejos necesario ni imperativo cambiar sus genitales, otras sí... Conozco a mujeres fabulosas y con pene, y hombres indiscutibles sin él... (Com53, Art1)

En términos generales, se asume que la necesidad de modificar el cuerpo responde a la presión social, que es producto de la forma en que en nuestra sociedad se vinculan género y cuerpo. En la siguiente cita, por ejemplo, se explicita que las personas trans no necesitarían la operación «si la gente fuera capaz de aceptarnos con los cuerpos con los que nacemos en el género que nos reconocemos» (Com71, Art1). A diferencia del discurso transexual, se considera que el sufrimiento por ser trans no tiene una causa individual, sino que está condicionado por el entorno: el rechazo al propio cuerpo y la necesidad de modificarlo no serían cuestiones innatas, sino que estarían relacionadas con el estigma social que implica vivir en un cuerpo no normativo.

En relación con la reproducción del binomio formado por hombre y mujer, el argumento más frecuente es que no se trata de excluir a quien se sienta cómodo con las categorías de mujer y hombre, sino de incluir también otras opciones: «Es reconocer que existe una multitud de formas, desde las más definidas hasta las más ambiguas» (Com27, Art1). Desde esta lógica integradora, 
se propone que la categoría trans funcione como término paraguas que incluya a todas las personas que no se sientan a gusto con el género asignado al nacer. Asimismo, se critica a las que, desde el discurso transexual, adoptan una actitud de «policía del género» (Com71, Art1, y Com61, Art1). Así, se cuestiona la misma distinción entre transexual y transgénero, por no ser realidades estancas y por no recoger la complejidad de las formas de vivir el género.

De la misma manera que encontramos posiciones autoritarias en el discurso transexual en el momento en que trata de imponerse una definición determinada de lo que es ser transexual, también aparece un tono autoritario en algunas intervenciones de personas que mantienen un discurso transgénero. En este sentido, una participante en el debate, por ejemplo, considera que las personas transexuales siguen «lo que marcan las masas borreguiles» y tilda a la autora del artículo 1 de «tradicionalista, beata talibán del binarismo y boicoteadora ignorante de lo más rancio y cutre de la sociedad» (Com40, Art1). Este autoritarismo se basa en distinguir entre una élite transgénero y queer que estaría en posesión de la verdad, en contraposición a unas personas transexuales que estarían anuladas y engañadas por parte del sistema patriarcal. Así se desprende de la siguiente cita:

Si tú quieres seguir siendo sumisa al poder, adelante con ello. Yo me estaré hormonando sin pasar por un loquero desde el primer día mientras tú explicarás que eres superwoman, superfemenina y superhetero. [...] unxs nos movemos por la libertad a sentir como sentimos y otrxs os movéis por favores políticos, sin más razón que conquistar la sumisión autoritariamente para ejercer el poder hacia tus iguales. (Com40, Art1)

Este tipo de argumentaciones, analizada con más detenimiento en CollPlanas (2010: 91 s.), reproduce una lógica según la cual hay unas personas (las transgénero y queer, en este caso) que son plenamente sujetos, en contraposición a otras personas que son puros objetos de intereses ajenos.

\section{Conclusiones}

En este artículo, hemos analizado las posiciones en el debate en torno al uso de las categorías transexual y transgénero, en el que se contraponen dos formas de entender el género, su conexión con el cuerpo, los objetivos políticos y las estrategias para alcanzarlos. En este debate, observamos dos posturas enfrentadas que se reproducen tanto a nivel individual como colectivo: el discurso transexual y el transgénero. En el discurso transexual, se establece como prioritaria la «necesidad» de realizar la transición, entendiendo que la motivación para cambiar de sexo es una determinación biológica, inmodificable y, por lo tanto, no elegida. En el discurso transgénero, aquello que se establece como prioritario es liberarse de las presiones de género a través de la crítica al modelo binarista y a la reproducción de los roles de género normativos.

¿A qué se debe este nivel de belicosidad en la defensa de las fronteras identitarias? ¿Qué hay detrás de este debate aparentemente terminológico? 
En primer lugar, una razón que explica la confrontación para establecer los límites de la categoría transexual es que estar emparado en ella supone ser reconocido a través de un diagnóstico, lo cual tiene importantes efectos prácticos: poder cambiar la identidad legal, hormonarse controladamente, operarse... Además, implica asumir un discurso que confiere un sentido a la experiencia vivida, desculpabiliza y ofrece la promesa de dejar de sufrir. La explicación que da la medicina acerca de las causas del trastorno de identidad de género es muy tranquilizadora para un gran número de personas, dado que las despoja de agencia en relación con su transexualidad y convierte el deseo de cambiar de sexo en algo legítimo e ineludible (Coll-Planas, 2010).

En segundo lugar, las tensiones que existen dentro de la comunidad trans para delimitar la categoría transexual tienen que ver con la búsqueda del reconocimiento social a través de una identidad reconocida y legitimada por la medicina.

Finalmente, toda identidad grupal se construye mediante la oposición o la exclusión a otros, fenómeno conocido como «narcisismo de las pequeñas diferencias»: «la positividad de nuestras identidades depende, en gran parte, del contraste, de la diferencia en relación con modelos que rechazamos o negamos» (Bezerra, 2000: 77) ${ }^{7}$. Así, toda identidad se construye y se reproduce en oposición a exteriores constitutivos: masculinidad/feminidad, heterosexualidad/ homosexualidad, homosexualidad/transexualidad, transexualidad/transgenerismo, transexualidad/travestismo, etc. Esta categorización implica la atribución de diferentes grados de legitimidad, reconocimiento y acceso a derechos. En el caso que nos ocupa, se produce una jerarquización de las trayectorias trans, estableciendo como más legítimas las que son sancionadas por el estamento médico, que es el que controla el acceso, por ejemplo, al control endocrinológico de la hormonación, al cambio de identidad legal o al permiso para intervenirse quirúrgicamente.

El discurso médico ha establecido criterios diagnósticos muy rígidos para identificar a una persona transexual, con lo cual ha guardado las fronteras de la categoría. La normatividad de dicha categoría ha sido muy efectiva, hasta el punto en que quienes patrullan sus límites ya no son solo las instituciones médicas, sino también, y en gran parte, las personas transexuales que se identifican con el discurso médico y cuya subjetividad está en parte configurada en relación con este (Coll-Planas y Missé, 2012).

Sin embargo, no es solo el discurso transexual el que establece una normatividad. Algunas formulaciones del discurso transgénero también crean una normatividad que excluye a las personas trans que desean operarse o reproducir la identidad de género estable. Así, el discurso transgénero, que emerge en oposición al discurso médico por considerarlo autoritario y no inclusivo, puede acabar reproduciendo las lógicas de exclusión que reprocha a la patologización de la transexualidad, al establecer una jerarquía entre personas críticas y personas reproductoras del género normativo.

7. Traducción de los autores. 


\section{Referencias bibliográficas}

Balza, I. (2009). «Bioética de los cuerpos sexuados: Transexualidad, intersexualidad y transgenerismo». Isegoría: Revista de Filosofía Moral y Politica [en línea], 40, 245-258. <http://dx.doi.org/10.3989/isegoria.2009.i40.658>.

Belsué, K. (2011). «Sexo, género y transexualidad: De los desafíos teóricos a las debilidades de la legislación española». Acciones e Investigaciones Sociales, 29, 7-32.

- (2012). «La legislación en torno a la transexualidad en España: Avances, debilidades y paradojas». Feminismo/s, 19, 211-234.

Bezerra, B. (2000). "Aids e os processos de construçao identitaria». En: Banco De Horas. Primeiro Simpósio Subjetividade e Aids: O Livro. Río de Janeiro: Banco de Horas / IDAC.

Billings, D. y Urban, T. (1983). «La construcción socio-médica de la transexualidad: interpretación y crítica». En: Nieto, José Antonio (comp.). Transexualidad, transgenderismo y cultura: Antropología, identidad y género. Madrid: Talasa, 1998.

Burr, V. (1996). Introducció al construccionisme social. Barcelona: Universitat Oberta de Catalunya / Proa.

Butler, J. (1990). El género en disputa: El feminismo y la subversión de la identidad. Paidós: Ciudad de México, 2001.

- (1997). Mecanismos psíquicos del poder: Teorías sobre la sujeción. Madrid: Cátedra, 2001.

- (2004). Deshacer el género. Barcelona: Paidós, 2006.

CABral, M. (ed.) (2009). Interdicciones: Escrituras de la intersexualidad en castellano. Córdoba: Anarrés.

Carratalá, A. (2011). «¿Smail o Cristina?: Desigualdad e identidad transexual en el discurso periodístico». Zer: Revista de Estudios de Comunicación / Komunikazio Ikasketen Aldizkaria, 31, 183-201.

Casanova, A. (2011). «Trans-formar la educación primaria: Reflexiones de un maestro transexual». Cuadernos de Pedagogía, 414, 84-86.

Coll-Planas, G. (2010). La voluntad y el deseo. La construcción social del género y la sexualidad: El caso de lesbianas, gays y trans. Barcelona/Madrid: Egales.

- (2012). La carne y la metáfora: Una reflexión sobre el cuerpo en la teoría queer. Barcelona/Madrid: Egales.

Coll-Planas, G.; Bustamante, G. y Missé, M. (2009). Transitant per les fronteres del gènere: Estratègies, trajectòries $i$ aportacions de joves lesbianes, gais i trans. Barcelona: Observatori Català de la Joventut.

Coll-Planas, G. y Missé, M. (2012). «Le genre et la chair: Analyse des discours médicaux autour de la transsexualité dans le cas espagnol». Nouvelles Questions Féministes, 31 (1), 60-79.

FeInberg, L. (1992). Transgender liberation: A movement whose time has come. Nueva York: World View Forum.

Fisher, H. (1999). El primer sexo: Las capacidades innatas de las mujeres y cómo están cambiando el mundo. Madrid: Taurus, 2000.

FlyvbJerg, B. (2004). «Cinco malentendidos acerca de la investigación mediante los estudios de caso». Reis: Revista Española de Investigaciones Sociológicas, 106, 33-62.

Fontana, S. y Ferriols Tierno, M. J. (2011). "Hablemos de diversidad sexual». Cuadernos de Pedagogía, 414, 87-90.

Foucault, M. (1976). Historia de la sexualidad. 1: La voluntad de saber. Madrid: Siglo XXI, 1995. 
Fuss, D. (1989). En essència: Feminisme, naturalesa i diferència. Vic: Eumo Editorial, 1999.

García, A. y Oñate, S. (2010). "De viajes y cuerpos: proyectos migratorios e itinerarios corporales de mujeres transexuales ecuatorianas en Murcia». En: García, A. A.; Gadea, M. E. y Pedreño, A. (eds.). Tránsitos migratorios: Contextos transnacionales y proyectos familiares en las migraciones actuales. Murcia: Universidad de Murcia, 361-384.

Goldberg, S. (1973). La inevitabilidad del patriarcado. Madrid: Alianza, 1976.

Hausman, B. (1998). "En busca de la subjetividad: Transexualidad, medicina y tecnologías de género». En: Nieto, José Antonio (comp.). Transexualidad, transgenderismo y cultura: Antropología, identidad y género. Madrid: Talasa.

Hinojosa, M. J. (2011). «Desarrollo de la identidad en lesbianas, gays, transexuales y bisexuales». Cuadernos de Pedagogía, 414, 30-33.

Juliano, M. D. (2004). Excluidas y marginales. Madrid: Cátedra.

- (2010). «El cuerpo fluido: Una visión transdiciplinar desde la antropología». Quaderns de Psicología, 12 (2), 149-160.

KING, D. (1998). "Confusiones de género: Concepciones psicológicas y psiquiátricas sobre el trasvestismo y la transexualidad». En: Nieto, José Antonio (comp.). Transexualidad, transgenderismo y cultura: Antropología, identidad y género. Madrid: Talasa.

Lozano, M. (2011). «Sexualidades enredadas». Cuadernos de Pedagogía, 414, 91-94.

Llamas, R. (1998). Teoría torcida: Prejuicios y discursos en torno a la «homosexualidad». Madrid: Siglo XXI.

Marini, L.; Medina, P. y Rodrigo, M. (2010). «Prensa on-line y transexualidad: Análisis de la cobertura periodística del caso de Thomas Beatie». Estudios sobre el Mensaje Periodístico, 16, 291-306.

Martínez, A. e Íñiguez, L. (2010). «La fabricación del Trastorno de Identidad Sexual: Estrategias discursivas en la patologización de la transexualidad». Discurso \& Sociedad, 4 (1), 30-51.

Martínez, A. y Montenegro, M. (2010). «Narrativas en torno al trastorno de identidad sexual: De la multiplicidad transgénero a la producción de trans-conocimientos». Prisma Social: Revista de Ciencias Sociales, 4, 3.

- (2011). «El desafío trans: Consideraciones para un abordaje situado de las identidades de sexo/género». Sociedad y Equidad: Revista de Humanidades, Ciencias Sociales, Artes y Comunicaciones, 2, 3-22.

Missé, M. (2013). Transexualidades: Otras miradas posibles. Madrid/Barcelona: Egales.

Missé, M. y Coll-Planas, G. (ed.) (2010). El género desordenado: Críticas en torno a la patologización de la transexualidad. Madrid/Barcelona: Egales.

Nieto, J. A. (2006). "Transexualidad: Sus supuestas causas innatas». Sistema: Revista de Ciencias Sociales, 193, 75-96.

PÉrez, K. (2010). «Historia de la patologización y despatologización de las variantes de género». En: Missé, M. y Coll-Planas, G. (eds.). El género desordenado: Críticas en torno a la patologización de la transexualidad. Barcelona/Madrid: Egales, 97-111.

Platero, R. (L.) (2007). «iMaricón el último!: Docentes que actuamos ante el acoso escolar». Revista d'Estudis de la Violència, 3, 2.

- (2009). «Transexualidad y agenda política: Una historia de (dis)continuidades y patologización». Política y Sociedad, 46, 107-128.

Rodríguez, R. (2001). «Análisis antropológico de la transexualidad, entre la realidad cultural y la resistencia social». Anuario de Filosofía, Psicología y Sociología, 4, 239-248. 
Rubio, F. J. (2008). «¿El tercer género?: La transexualidad». Nómadas: Revista Crítica de Ciencias Sociales y Juridicas, 17, 47-54.

Soley-Beltran, P. (2005). «In-transit: La transexualidad como migración de género». Asparkia: Investigación feminista, 15, 207-32.

- (2007). «Transsexualism in Spain: a Cultural and Legal Perspective». Sociological Research Online [en línea], 12 (1). $<$ http://dx.doi.org/10.5153/sro.1474>.

- (2009). Transexualidad y la matriz heterosexual: Un estudio critico de Judith Butler. Barcelona: Bellaterra.

Soley-Beltran, P. y Coll-Planas, G. (2011). "Having Words for Everything”: Institutionalising Gender Migration in Spain (1998-2008)». Sexualities, 3 (14), 334-353. <http://dx.doi.org/10.1177/1363460711400811>.

Stone, S. (1991). "The Empire Strikes Back: A Posttranssexual Manifesto». Body Guards: The Cultural Politics of Gender Ambiguity. Nueva York: Routledge, 280-304.

Stryker, S. (2008). Transgender history. Berkeley: Seal Press.

Tena, F. (2013). «Sacudirse la tutela médica: Hacia la despatologización de la transexualidad». Revista Andaluza de Antropología, 5, 35-65.

Valentine, D. (2007). Imagining transgender: An ethnography of a category [en línea]. Durham: Duke University Press. <http://dx.doi.org/10.1215/9780822390213>.

VARTABEDIAN, J. (2007). «El cuerpo como espejo de las construcciones de género: Una aproximación a la transexualidad femenina». Quaderns-E de l'ICA, 10, 3.

- (2008). «Transexualidad femenina en Barcelona: Notas sobre un trabajo de campo». (con)textos: Revista d'Antropologia i Investigació Social, 1, 107-114.

- (2012). Geografia travesti: Cuerpos, sexualidad y migraciones de travestis brasileñas (Rio de Janeiro-Barcelona). Universitat de Barcelona. Tesis doctoral.

Weeks, J. (1981). Sex, Politics \& Society: The regulation of sexuality since 1800. Londres: Longman.

- (1985). El malestar de la sexualidad: Significados, mitos y sexualidades modernas. Madrid: Talasa, 1993. 